\title{
Epidemiología de la Urgencia Psiquiátrica en el Instituto Psiquiátrico "Dr. José Horwitz": Un nuevo estudio comparativo Epidemiology of Psychiatric Emergency Consultations Psychiatric Institute "Dr. José Horwitz": A new comparative study
}

\author{
Carlos Cruz M..$^{1}$, Jorge Leiva L. ${ }^{2}$, Rafael Borgoño V. ${ }^{3}$, Tomás Larach K. ${ }^{3}$, \\ Loreto Errázuriz B. ${ }^{3}$, Ignacio Chianale C. ${ }^{3}$, Astrid Bown S. ${ }^{3}$ y Alejandro Trapp U. ${ }^{4}$
}

\begin{abstract}
Introduction: A psychiatric emergency is any alteration of affection, thought or behavior that may cause harm to others or to the same consultant. These consultations have increased over the last years. Objectives: Determine the prevalence of the various pathologies, describing their distribution according to sex, age, area of residence, marital status, occupation, health insurance, and referral in psychiatric emergency consultations at "Dr. José Horwitz" Hospital of Santiago, and to compare these results with previously published studies in the same center. Material and Method: We used a representative sample of 1999 cases, chosen randomly from consultations held between July 1, 2005 and June 30, 2006. Diagnoses were grouped according to the nomenclature of the ICD-10. Results: $48.7 \%$ of the consultations were carried out by male patients and. Average age of consultation was 39. $76.7 \%$ of consultations were carried out in daytime. The $48.3 \%$ of people who consulted didn't have any occupation. $48 \%$ were singles. Abuse of substances and alcohol, schizoid, afective and neurotic disorders congregate $73.9 \%$ of total consultations. The most prevalent were the disorders associated with abuse of substances and alcohols with a $19.7 \%$ overall. Autoagressive behaviors (74\%) and affective disorders (74\%) were associated with females, while abuse of substances and alcohol was associated with males (76\%). Discussion: Schizoid disorders have proportionally declined in importance over the years. In contrast, abuse of substances and alcohol, and affective disorders have increased their frecuency. Suggestions are made to FACE the new epidemiological profile.
\end{abstract}

Key words: Psychiatric emergence, epidemiology, crisis intervention.

Rev Chil Neuro-Psiquiat 2010; 48 (3): 175-183

Recibido: 02/12/2009

Aceptado: 26/07/2010

Médico Psiquiatra, Servicio de Urgencia, Instituto Psiquiátrico Dr. José Horwitz B. Profesor Asociado Universidad Adolfo Ibáñez. Profesor Asistente Universidad Andrés Bello.

2 Médico Psiquiatra, Servicio de Urgencia, Instituto Psiquiátrico Dr. José Horwitz B.

3 Interno de Medicina, Universidad de los Andes.

4 Bioestadístico, Universidad de los Andes. 


\section{Introducción}

Se considera una urgencia psiquiátrica cualSquier alteración del afecto, de la conducta o del pensamiento que puede producir daños a terceros o al mismo consultante, por lo cual requiere de un diagnóstico inmediato para una intervención rápida y eficaz, dirigida a aliviar la situación inmediata ${ }^{1}$.

La consulta psiquiátrica de urgencia ha ido en aumento, lo que se atribuye a factores tan variados como la masificación y sectorización de la medicina, la crisis de valores occidentales, la divulgación del concepto de enfermedad mental, los avances en terapias, la desinstitucionalización de los enfermos mentales, etc ${ }^{2}$.

En un estudio previo realizado por F. Schürch en 1985 en el Instituto Psiquiátrico detectó que había un predominio de adultos jóvenes, de igual proporción por género, siendo los diagnósticos más frecuentes las neurosis y psicosis, clasificados según criterio sindromático ${ }^{3}$.

En un estudio que comparó la epidemiología de las consultas de Urgencia Psiquiátrica del Hospital Psiquiátrico de Santiago en los años 1988 y 1994, se evidenció que las psicosis eran la principal causa de consulta. Se vio una disminución de éstas, además de las neurosis y epilepsia, en relación a un aumento en la frecuencia de desórdenes afectivos y consultas por abuso de alcohol y drogas ${ }^{4}$. Entre otros resultados del mismo estudio, destaca un aumento de las consultas en horario nocturno, y un envejecimiento de la población consultante.

En una investigación reciente realizada por nuestro equipo, evaluamos la frecuencia de urgencias psiquiátricas, siguiendo los criterios CIE- $10^{5}$, en un hospital general y apreciamos que $52 \%$ de los pacientes eran mujeres; $48 \%$ eran menores de 35 años.

En términos diagnósticos, destacaron los trastornos por alcohol y drogas (32,7\%) y los trastornos neuróticos $(51,1 \%)$. En los hombres predominaron los trastornos por alcohol y drogas $(58,2 \%)$ y los trastornos neuróticos $(31,9 \%)$ de los cuales los trastornos somatomorfos eran el 62,8\%; mientras que en las mujeres fueron los trastornos neuróticos $(68,7 \%)$, los trastornos por alcohol y drogas $(9,3 \%)$ y las autolesiones intencionales $(10 \%)^{6}$.

Estos resultados coinciden con un estudio español, en el cual las consultas fueron evaluadas por psiquiatra. Se encuentran una paridad por género, siendo los motivos de consulta más frecuentes ansiedad, consumo de sustancias psicoactivas, depresión y alteraciones conductuales ${ }^{7}$.

El objetivo de este trabajo es determinar la prevalencia de las distintas patologías en las consultas de urgencia psiquiátrica en el Instituto Psiquiátrico de Santiago. Además, se busca describir la distribución de dichas patologías según sexo, edad, sector de residencia, estado civil, previsión, horario de atención y diagnóstico.

Se plantea como objetivo también, comparar la prevalencia de las patologías y la consulta según el grupo etario en relación a estudios de años anteriores realizados en la misma institución y sugerir estrategias de abordaje para el nuevo perfil epidemiológico de las urgencias.

\section{Material y Método}

El Servicio de Urgencias del Instituto Psiquiátrico de Santiago atiende alrededor de 20.000 consultas anuales. Para efectos de este estudio, seleccionamos una muestra representativa de 1999 casos (10\%), escogidos aleatoriamente de las consultas realizadas entre el $1^{\circ}$ de julio del 2005 y el 30 de junio de 2006.

Los antecedentes se obtuvieron a partir de los formularios de atención que quedan registrados en los archivos de dicho centro, en forma de planilla electrónica. Del formulario se obtuvieron las siguientes variables: sexo, edad, sector de residencia, estado civil, previsión, horario de atención y diagnóstico.

Los diagnósticos se agruparon de acuerdo a la nomenclatura del CIE-10 en:

1. Patología Médica.

2. Trastornos Orgánicos.

3. Abuso de Sustancias y Alcohol.

4. Trastornos Esquizoides. 
5. Trastornos Afectivos.

6. Trastornos Neuróticos.

7. Trastornos de la Conducta Asociados a Alteraciones Fisiológicas y Factores Físicos.

8. Trastornos de Personalidad.

9. Retardo Mental.

10. Trastornos del Desarrollo.

11. Conductas Autoagresivas.

Los datos se incluyeron dentro de una planilla en el programa Excel de Microsoft Office 2003, se separaron según diagnóstico clínico, graficando algunos resultados. Se utilizó el programa SPSS versión 12,5 para las pruebas estadísticas.

\section{Resultados}

De todas las consultas de urgencia estudiadas, $51,3 \%$ eran solicitadas por pacientes de sexo femenino.

La distribución por edad muestra un 51,6\% de consultas en pacientes entre 26 y 45 años. La media fue de 39 años (Tabla 1).

Del total de consultas, el 76,7\% fueron realizadas en horario diurno (8:00-19:59 hrs) El 99,2\% de los consultantes proviene de la Región Metropolitana y al distribuirlos por áreas de Salud se aprecia que el 55,3\% es Norte, $18 \%$ de Central, $14 \%$ de Occidente y $5 \%$ de Oriente.

La distribución según previsión muestra una concentración de pacientes beneficiarios de FONASA tipo A y B, tarjeta de gratuidad y pensión asistencial (81,6\%). Sólo el 2,9\% de las consultas corresponden al sector privado (Tabla 2).

En cuanto a la ocupación de la población estudiada, se observa que el $48,3 \%$ de éstos señala estar cesante al momento de consultar. El 60,2\% dice tener estudios de enseñanza media, técnica o superior completa (Figuras 1 y 2 ).

La distribución de la población consultante según estado civil, muestra que el mayor porcentaje $(48 \%)$ corresponde a personas solteras, $10,1 \%$ convive, $27,6 \%$ esta casado y el $9,6 \%$ se encuentra separado.

Al observar los datos relacionados con la derivación de los pacientes después de la consulta de urgencia, un 45,2\% de los ellos son derivados a consultorios periféricos, mientras que un $10 \%$ de éstos se hospitalizan en el Servicio.

$\mathrm{Al}$ analizar la distribución de las consultas según la clasificación CIE-10, se observa que las cuatro categorías más prevalentes (abuso de sustancias $\mathrm{y}$ alcohol, trastornos esquizoides, trastornos afectivos y trastornos neuróticos) muestran diferencias porcentuales pequeñas entre ellas y congregan el

Tabla 1. Distribución de la muestra según rango de edad

\begin{tabular}{|c|c|c|c|c|c|c|}
\hline \multicolumn{7}{|c|}{ Edad } \\
\hline$\leq 25$ & $26-35$ & $36-45$ & $46-55$ & $56-65$ & $\geq 66$ & Total \\
\hline 353 & 504 & 528 & 364 & 167 & 83 & 1999 \\
\hline $17,70 \%$ & $25,20 \%$ & $26,40 \%$ & $18,20 \%$ & $8,40 \%$ & $4,20 \%$ & $100 \%$ \\
\hline
\end{tabular}

Tabla 2. Distribución de los consultantes según previsión

\begin{tabular}{|ccccc|}
\hline & \multicolumn{3}{c|}{ Previsión } \\
Fonasa A-B y otros $\left(^{*}\right)$ & Fonasa C-D & Isapre / Privado & Desconocidos & Total \\
1.631 & 298 & 57 & 13 & 1.999 \\
$81,60 \%$ & $14,90 \%$ & $2,90 \%$ & $0,70 \%$ & $100,00 \%$ \\
\hline
\end{tabular}


$73,9 \%$ del total de consultas, distribuyéndose el otro $26,1 \%$, en las 6 categorías restantes. La categoría diagnóstica más prevalente fue la de trastornos asociados al consumo de alcohol y drogas con un $19,7 \%$ del total (Tabla 3 ).

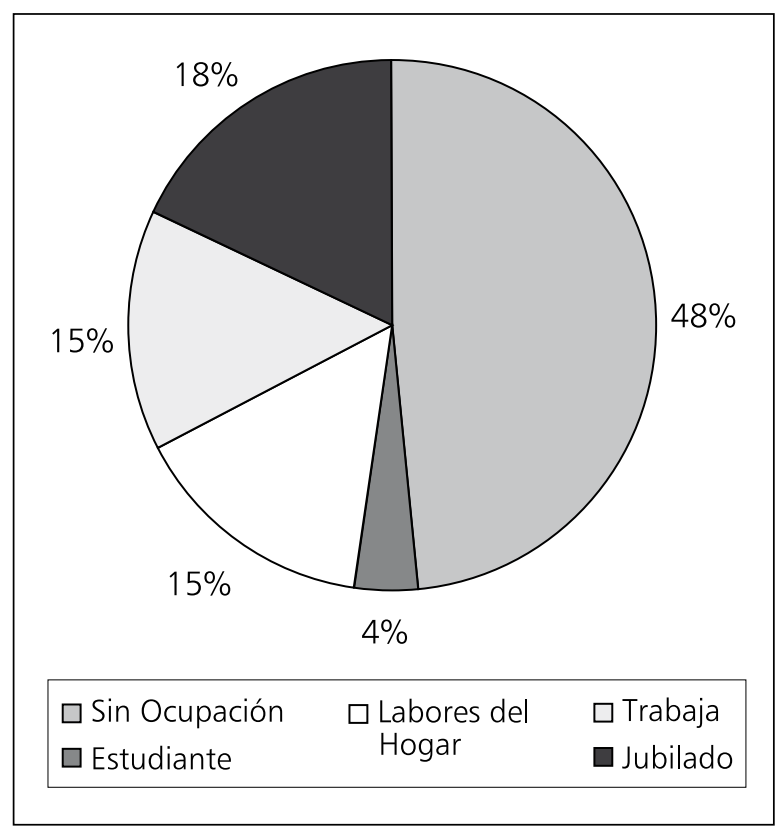

Figura 1. Distribución de la población consultante según actividad laboral.
Todas las categorías diagnósticas CIE-10 utilizadas presentan asociaciones significativas relacionadas con el género de los consultantes $(\mathrm{p}<0,0001)$ excepto la categoría "Patología Médica y Otros".

La asociación más significativa para el sexo

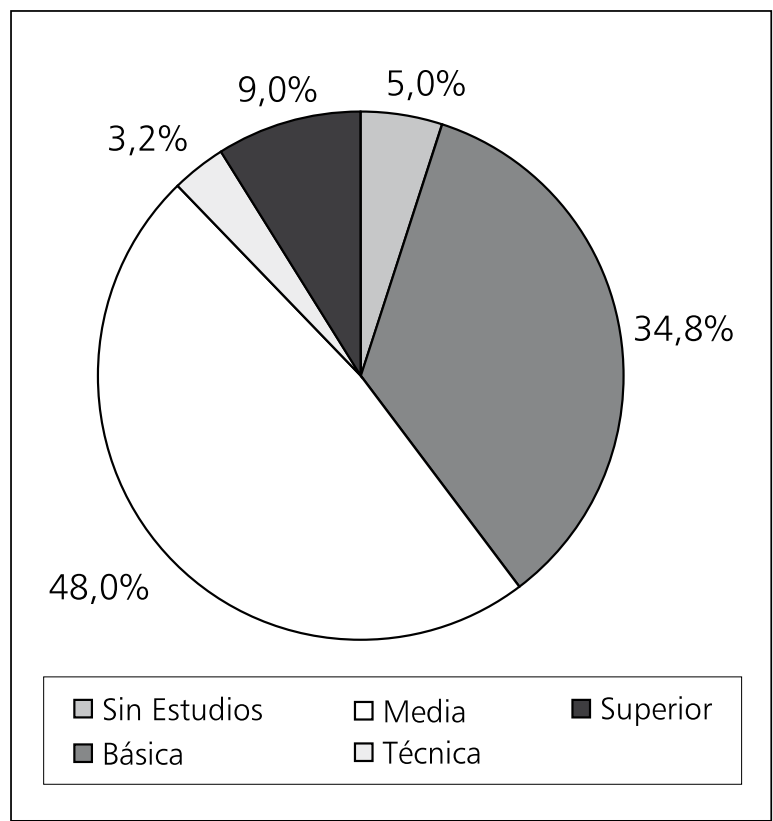

Figura 2. Distribución de los consultantes según escolaridad.

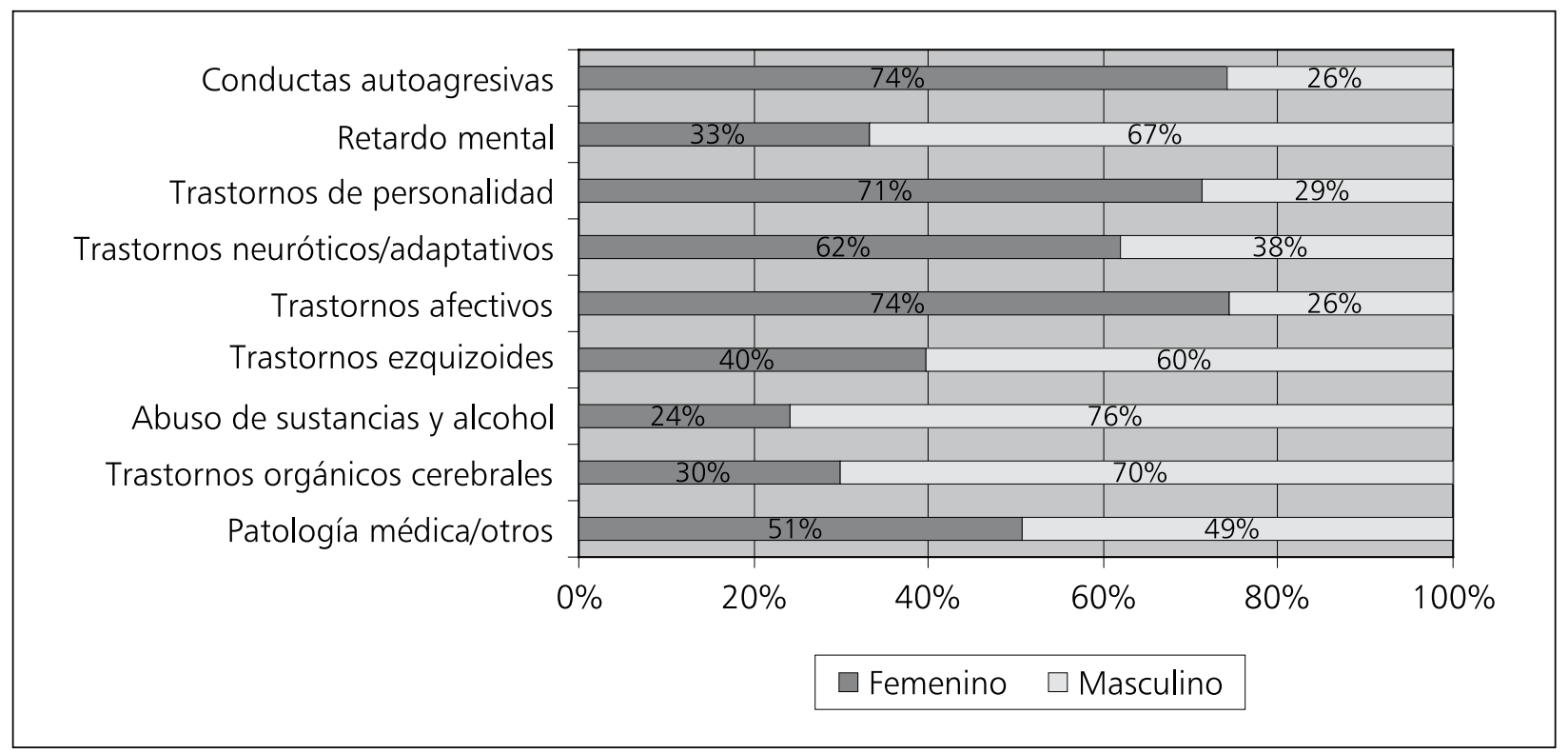

Figura 3. Distribución de sexo según categoría diagnóstica. 
masculino es con el abuso de sustancias y alcohol (76\%). Las conductas autoagresivas (74\%), trastornos de personalidad (71\%) y trastornos afectivos (74\%) son más frecuentes en pacientes de sexo femenino.

Los grupos con mayor porcentaje de pacientes jóvenes $(<35$ años) son las conductas autoagresivas y el retardo mental, ambos con 58\% (Tabla 4 ).

Tabla 3. Distribución del total de las consultas según el diagnóstico CIE-10

\begin{tabular}{|lcc|}
\hline Diagnóstico CIE-10 & Recuento & $\begin{array}{c}\% \text { del } \\
\text { total }\end{array}$ \\
\hline Patología médica/otros $\left.{ }^{*}\right)$ & 134 & $6,7 \%$ \\
$\begin{array}{l}\text { Trastornos orgánicos } \\
\text { cerebrales }\end{array}$ & 70 & $3,5 \%$ \\
$\begin{array}{l}\text { Abuso de sustancias y } \\
\text { alcohol }\end{array}$ & 394 & $19,7 \%$ \\
Trastornos ezquizoides & 381 & $19,1 \%$ \\
Trastornos afectivos & 367 & $18,4 \%$ \\
$\begin{array}{l}\text { Trastornos neuróticos/ } \\
\text { adaptativos }\end{array}$ & 334 & $16,7 \%$ \\
\hline Trastornos de personalidad & 171 & $8,6 \%$ \\
Retardo mental & 51 & $2,6 \%$ \\
\hline Conductas autoagresivas & 97 & $4,9 \%$ \\
\hline Total & 1.999 & $100 \%$ \\
\hline
\end{tabular}

Todas las consultas, independiente de la categoría CIE-10, se presentan mayoritariamente durante el día. El mayor porcentaje de consultas nocturnas (29\%) esta dado por los trastornos neuróticos/ adaptativos. La categoría con mayor prevalencia de consultas diurnas es abuso de sustancias y alcohol, con un $82 \%$ de los casos.

Se destaca que el $76 \%$ de los pacientes con conductas auto agresivas completaron a lo menos enseñanza media, constituyendo el grupo con nivel educativo más alto. Las categorías con menor porcentaje de pacientes con estudios superiores 0 técnicos esta dado por aquellos con retraso mental $(0,1 \%)$, patología medica (6\%), abuso de sustancias $\mathrm{y}$ alcohol, y trastornos esquizoides (ambos con $10 \%)$.

Todas las categorías presentan un porcentaje menor al 5\% de estudiantes, salvo el grupo de conductas autoagresivas en donde el $12 \%$ corresponde a éstos. Los grupos con porcentajes más altos de cesantes se encuentran en los con retraso mental (92\%), trastornos esquizoides $(85 \%)$ y abuso de sustancias y $\mathrm{OH}(74 \%)$.

Los pacientes con retardo mental son los que menos se casan (88\%), seguido de los trastornos esquizoides $(72 \%)$. Los grupos con mayor porcentaje de viudos son los trastornos afectivos y los trastornos orgánicos cerebrales, ambos con un $7 \%$.

Tabla 4. Distribución de edad por categoría diagnóstica

\begin{tabular}{|c|c|c|c|c|c|c|}
\hline Diagnóstico CIE-10 & $\leq 25$ & 26-35 & $36-45$ & $46-55$ & $56-65$ & $\geq 66$ \\
\hline Patología médica/otros & $18 \%$ & $22 \%$ & $23 \%$ & $20 \%$ & $9 \%$ & $8 \%$ \\
\hline Trastornos orgánicos cerebrales & $7 \%$ & $16 \%$ & $23 \%$ & $11 \%$ & $14 \%$ & $29 \%$ \\
\hline Abuso de sustancias y alcohol & $19 \%$ & $26 \%$ & $26 \%$ & $21 \%$ & $8 \%$ & $1 \%$ \\
\hline Trastornos ezquizoides & $17 \%$ & $25 \%$ & $30 \%$ & $16 \%$ & $9 \%$ & $2 \%$ \\
\hline Trastornos afectivos & $15 \%$ & $22 \%$ & $23 \%$ & $23 \%$ & $12 \%$ & $5 \%$ \\
\hline Trastornos neuróticos / adaptativos & $17 \%$ & $31 \%$ & $25 \%$ & $18 \%$ & $4 \%$ & $4 \%$ \\
\hline Trastornos de personalidad & $15 \%$ & $26 \%$ & $37 \%$ & $15 \%$ & $6 \%$ & $1 \%$ \\
\hline Retardo mental & $27 \%$ & $31 \%$ & $31 \%$ & $6 \%$ & $4 \%$ & $0 \%$ \\
\hline Conductas autoagresivas & $33 \%$ & $25 \%$ & $20 \%$ & $13 \%$ & $7 \%$ & $2 \%$ \\
\hline
\end{tabular}




\section{Discusión}

Uno de los principales problemas encontrados durante la recopilación y análisis de los datos fue la "anarquía terminológica" con que se manejan las hipótesis diagnósticas en las fichas de los pacientes. La explicación más plausible para esto es la variedad de médicos y personal que atiende en el Servicio de Urgencias durante el período de un año, y que no necesariamente utilizan la nomenclatura del DSM-IV o CIE-10 para consignar sus diagnósticos. Algunas fichas de las seleccionadas al azar no tenían una hipótesis diagnóstica estipulada, por lo que para realizar la clasificación fue necesario guiarse por el diagnóstico sindromático o antecedentes del paciente y las alteraciones de la conducta. Además, al no encontrar motivo de consulta en las fichas, se debió priorizar el hecho "más agudo" encontrado en la hipótesis diagnóstica o el diagnóstico sindromático, para establecer un diagnóstico único dentro de la clasificación del CIE-10. Los futuros estudios deberán atenerse a clasificaciones internacionales para establecer comparaciones válidas.

Es posible observar de los datos obtenidos que más de la mitad de las consultas son de personas provenientes del área de Salud Norte de la capital. Esto se explica por la ubicación de este recinto hospitalario, y no necesariamente por un aumento de la patología psiquiátrica en este grupo poblacional. Observamos un fenómeno similar al estudiar las urgencias psiquiátricas en la asistencia pública ${ }^{6}$.

Comparamos nuestros resultados con los obtenidos en años anteriores y que fueron analizados por Escobar et $\mathrm{al}^{4}$. En este estudio compararon los datos recopilados en este mismo establecimiento durante los años 1988 y 1994 (Tabla 5).

Del análisis de la Tabla 6 se puede decir que la consulta en menores de 34 años ha disminuido a lo largo de los años, mientras que ha habido un aumento relativo importante de la consulta en el grupo de entre 35 y 54 años de edad. Los mayores de 55 años tienden a permanecer relativamente estables en sus porcentajes.

De la Tabla 7, por otra parte, se puede apreciar el aumento en la prevalencia del consumo de alco- hol y drogas, que prácticamente se duplica respecto al año 1988. Ocurre lo mismo con los trastornos afectivos, cuya prevalencia se triplica.

Se observa como han disminuido en importancia, proporcionalmente, los trastornos ezquizoides a lo largo de los años y es probable que estos cambios en la prevalencia de los diagnósticos, afecte la distribución de las consultas según la edad en comparación a años anteriores, ya que la mayoría de los casos de consumo de alcohol y drogas, y de los trastornos afectivos, se concentran en los grupos etáreos intermedios. Estos datos concuerdan con los estudios que muestran un aumento relativo en la prevalencia de los trastornos afectivos y el consumo de alcohol y drogas en hospitales generales ${ }^{6,7}$.

Sin embargo, cabe decir que hemos encontrado pocos estudios referidos a la prevalencia de trastornos en Urgencias psiquiátricas especializadas. Mencionaremos el de González-Juárez en España ${ }^{8}$ que observó frecuencias de psicosis, trastornos afectivos y neuróticos similares a los nuestros, pero con un porcentaje menor de abuso de sustancias (9,7\%).

Por otra parte, debemos considerar el aumento sostenido de los trastornos por abuso de sustan-

Tabla 5. Comparación de consultantes por grupo de edad en los diferentes estudios

\begin{tabular}{|ccccc|}
\hline Año & \multicolumn{5}{c|}{ Edad } & & \\
& $\leq \mathbf{3 4}$ & $\mathbf{3 5 - 5 4}$ & $\geq \mathbf{5 5}$ & No registrados \\
\hline 1988 & $57,2 \%$ & $26,8 \%$ & $11,2 \%$ & $4,8 \%$ \\
1994 & $47,9 \%$ & $37,2 \%$ & $14,9 \%$ & \\
2006 & $39,6 \%$ & $47,3 \%$ & $13,1 \%$ & \\
\hline
\end{tabular}

Tabla 6. Comparación de consultantes por diagnóstico en los diferentes estudios

\begin{tabular}{|lrrr|}
\hline Diagnósticos & \multicolumn{3}{c|}{ Año } \\
& $\mathbf{1 9 8 8}$ & $\mathbf{1 9 9 4}$ & $\mathbf{2 0 0 6}$ \\
\hline Trastornos ezquizoides & $31,6 \%$ & $26,2 \%$ & $19,1 \%$ \\
Alcohol y drogas & $10,0 \%$ & $17,9 \%$ & $19,7 \%$ \\
Trastornos neuróticos & $21,2 \%$ & $11,9 \%$ & $16,7 \%$ \\
Trastornos afectivos & $5,80 \%$ & $15,80 \%$ & $18,40 \%$ \\
Otros & $31,40 \%$ & $28,20 \%$ & $26,10 \%$ \\
\hline
\end{tabular}


cias psicoactivas, con predominio del alcohol ${ }^{9}$, generalmente asociado a comorbilidad tales como ansiedad y distimia, y a conductas tanto auto como heteroagresivas, como también la predominancia de los trastornos afectivos observado en éste y otros estudios ${ }^{8}$.

Estos resultados permiten sugerir algunas estrategias que un Servicio de Urgencias Psiquiátricas debiera adoptar:

- Homogenizar la recopilación de datos clínicos del paciente con el uso del CIE-10 y reducir las variables a completar, siguiendo un criterio clínico y de utilidad del dato para investigar procesos diagnósticos y terapéuticos.

- Es importante precisar la verdadera urgencia de la consulta y determinar si los pacientes son repetidores o desean la obtención de fármacos como motivo principal de la consulta, dato que no se menciona en este estudio.

- El manejo de este nuevo perfil epidemiológico de la población atendida en el Servicio, debiera ir acompañado de la revisión, no sólo de los procedimientos, sino de una actualización de un arsenal farmacológico para este tipo de pacientes. Ya disponemos de criterios internacionales para nuestras intervenciones ${ }^{10,11,12}$

En éstas se enfatiza la necesidad de enfrentar adecuadamente las alteraciones conductuales, en especial la agresión y el uso juicioso de fármacos para su control, teniendo presente ante todo la seguridad del paciente ${ }^{13}$.
- Adicionalmente, la implementación en Chile de las Garantías Explícitas en Salud (GES) en Psiquiatría (esquizofrenia, depresión, alcohol y drogas) obliga a conocer sus indicaciones y reglamentación para trabajar de acuerdo a ellas ${ }^{14-17}$.

- La evolución del tipo de consultantes y su diagnóstico indica la reorientación que debiera tener el servicio en términos de las prestaciones que ofrecerá, en especial en lo referente al abuso de alcohol y drogas, las autoagresiones y las descompensaciones emocionales. Intervenciones previas realizadas por C. Martínez y su equi$\mathrm{po}^{18-20}$ tanto en hospital general como en este Servicio de Urgencias, han demostrado resultados muy esperanzadores para los terapeutas y para los pacientes. Estudios internacionales apoyan estos hallazgos ${ }^{21-23}$.

- Finalmente, un nuevo movimiento esta en desarrollo, el de considerar más a fondo las expectativas y preferencias de los pacientes, no en términos de cómo negociar un tratamiento efectivo y basado en la evidencia, sino teniendo en cuenta lo que el paciente desea y su impacto en la calidad de vida ${ }^{12,24}$.

$\mathrm{Al}$ aplicar por primera vez una encuesta de satisfacción usuaria en el Servicio hemos descubierto que la infraestructura y un trato amable son las dos variables que más determinan la satisfacción del paciente (C. Cruz, estudio enviado a publicación). 


\begin{abstract}
Resumen
Introducción: Una urgencia psiquiátrica es cualquier alteración del afecto, conducta o pensamiento que puede producir daños a terceros o al consultante. La consulta de urgencia ido en aumento en los últimos años. Objetivos: Determinar la prevalencia de las distintas patologías, describir su distribución según sexo, edad, sector de residencia, estado civil, ocupación, previsión y derivación en las consultas de urgencia psiquiátrica en el Hospital Psiquiátrico "Dr. José Horwitz" de Santiago y comparar estos resultados con publicaciones previas de estudios en el mismo centro. Material y Método: Muestra representativa de 1999 casos, escogidos aleatoriamente de las consultas realizadas entre el $1^{\circ}$ de julio de 2005 y el 30 de junio de 2006. Se agruparon los diagnósticos de acuerdo a la nomenclatura CIE-10. Resultados: 48,7\% de las consultas fueron realizadas por pacientes de sexo masculino. La media de edad fue 39 años. 76,7\% de las consultas fueron realizadas en horario diurno. El 48,3\% de los pacientes no tiene ocupación. $48 \%$ eran solteros. Abuso de sustancias y alcohol, trastornos esquizoides, afectivos y neuróticos congregan el 73,9\% de las consultas. La más prevalente fue la de trastornos asociados al abuso de sustancias $y$ alcohol con un 19,7\% del total. Conductas autoagresivas (74\%) y trastornos afectivos (74\%) se asoció más a mujeres, en cambio el abuso de sustancias y alcohol más con hombres (76\%). Discusión: Las consultas por trastornos esquizoides han disminuido a lo largo de los años. En cambio las consultas por el abuso de sustancias y alcohol y los trastornos afectivos han aumentado. Se hacen sugerencias para enfrentar el nuevo perfil epidemiológico.
\end{abstract}

Palabras clave: Urgencia psiquiátrica, epidemiología, intervención en crisis.

\section{Referencias}

1. Weil K. Psiquiatría. En: Florenzano R, Carvajal C, Weil K, editores. Santiago, Chile: Editorial Mediterráneo 1999; 216-27.

2. Escobar E. Urgencias Psiquiátricas. Primera Edición, Santiago de Chile, Sociedad de Neurología, Psiquiatría y Neurocirugía.

3. Schürch F. Características de la población consultante a un servicio de urgencia psiquiátrica. Rev Chil Neuro-Psiquiat 1985; 23: 29-38.

4. Escobar E, Kirschbaum A, Riquelme L, Schürch F, Núñez C. Demanda de consultas al Servicio de Urgencia del Instituto Psiquiátrico “Dr. José Horwitz B.” de Santiago: comparación entre 1988 y 1994. Rev Med Chile 1997; 125: 446-50.

5. CIE-10. Trastornos mentales y del comportamiento: descripciones clínicas y pautas para el diagnóstico. OMS: Editorial Meditor, 1992.

6. Cruz C, Leiva J, Escobar E, Eugenín D, Errázuriz $\mathrm{M}$, Prieto B, et al. Demanda espontánea de urgen- cias psiquiátricas al Hospital Asistencia Pública Dr. Alejandro del Río. Rev Chil Neuro-Psiquiat 2007; 45: 108-13.

7. Rabassó J, Parramon G, Goikolea J, Corbella B, Fuente E. Complejidad y adecuación de las urgencias psiquiátricas en un hospital general. Med Clin (Barc) 2003; 121: 650-52.

8. González-Juárez C, Pérez-Pérez E, Morales J, Morales M, Tierno R, Varela S. Factores asociados con la resolución de las urgencias psiquiátricas. Rev Asoc Esp Neuropsiq 2008: 28: 27-41.

9. Lejoyeux M, Boulenguiez S, Fichelle A, Mcloughlin $\mathrm{M}$, Ades J. Alcohol dependence among patients admitted to psychiatric emergency services. Gen Hosp Psychiatry 2000; 22: 206-12.

10. Climent C. Guía de práctica clínica basada en evidencias Urgencias Psiquiátricas. Versión en HTML disponible en: www.medynet.com/usuarios/jraguilar/Urgencias\%20Psquiatricas.pdf

11. Allen M, Currier G, Hughes D, Docherty J, Carpenter D, Ross R. Treatment of behavioural 
emergences: a summary of the Expert Consensus Guidelines. J Psychiatr Pract 2003; 9: 16-38.

12. Allen M, Currier G, Carpenter D, Ross R, Docherty J. Treatment of behavioural emergences 2005 . J Psychiatr Pract 2005; 11: 5-108.

13. Borckardt J, Grubaugh A, Pelic C, Kmett C, Hardesty S, Fruer C. Enhancing patient safety in psychiatric settings. J Psychiatr Pract 2007; 13: 355-61.

14. OMS-MINSAL. Informe WHO-AIMS sobre Sistema de Salud Mental en Chile, Santiago Chile 2006.

15. MINSAL (2006); Guía Clínica Tratamiento de Personas con Depresión Garantías Explícitas en Salud; Ministerio de Salud [on line] 2006, [citado en 2006-10-13] disponible en http://www.minsal. cl/ici/guias_clinicas_ges2006/DEPRESIONFIN.pdf

16. MINSAL (2005); Guía Clínica Primer Episodio Esquizofrenia Garantías Explícitas en Salud; Ministerio de Salud [on line] 2005, [citado en 200610-13] disponible en http://www.minsal.cl/ici/ guiasclinicas/EsquizofreniaR_Mayo10.pdf

17. MINSAL (1999); Plan Nacional de Salud Mental y Psiquiatría (2000-2010); Ministerio de Salud [on line] 2000, [citado en 2006-09-20) disponible en www.minsal.cl

18. Martínez C, Alonso C, Álvarez P, Oviedo C, Castro
P, Ellwanger J. Atención psicológica en un servicio de urgencia de hospital general. Revista de Psiquiatría y Salud Mental 2004; 21: 5-13.

19. Martínez C, Alonso C. La técnica de intervención en crisis en el Servicio de Urgencias del Instituto Psiquiátrico Dr. José Horwitz Barak. Revista de Psiquiatría y Salud Mental 2001; 18: 12-21.

20. Martínez C. Intervención en crisis en los 150 años del Instituto Psiquiátrico: una oportunidad para el cambio. Revista de Psiquiatría y Salud Mental 2003; 20: 208-15.

21. Catalano R, Mcconnell W, Forster P, Mcfarland B, Thornton D. Psychiatric emergency services and the system of care. Psychiatr Services 2003; 54: 351-5.

22. Hafner H, Rossler W, Haas S. Psychiatric emergency care and crisis intervention concepts, experiences and results. Psychiatr Prax 1986; 13: 203-12.

23. Schnyder U, Klaghofer R, Leuthold A, Buddeberg C. Characteristics of psychiatric emergencies and the choice of intervention strategies. Acta Psychiatr Scan 1999; 99: 179-87.

24. Murray K, Gary N. The next step in guideline development. Incorporating patients preferences. JAMA 2008; 4: 436-38.
Correspondencia:

Carlos Cruz M.

Luis Thayer Ojeda Norte 0130 oficina 607

Providencia.

Fono/Fax: 3661817.

E-mail: carloscruzmar@hotmail.com 